Trinity University

Digital Commons @ Trinity

Physics and Astronomy Faculty Research

Physics and Astronomy Department

$1-2019$

\title{
LIGO Analogy Lab-A Set of Undergraduate Lab Experiments to Demonstrate Some Principles of Gravitational Wave Detection
}

Dennis Ugolini

Trinity University, dugolini@trinity.edu

Hanna Rafferty

Trinity University, hraffert@trinity.edu

M. Winter

C. Rockstuhl

A. Bergmann

Follow this and additional works at: https://digitalcommons.trinity.edu/physics_faculty

Part of the Physics Commons

\section{Repository Citation}

Ugolini, D., Rafferty, H., Winter, M., Rockstuhl, C., \& Bergmann, A. (2019). LIGO analogy lab-A set of undergraduate lab experiments to demonstrate some principles of gravitational wave detection. American Journal of Physics, 87(1), 44-56. doi: 10.1119/1.5066567

This Article is brought to you for free and open access by the Physics and Astronomy Department at Digital Commons @ Trinity. It has been accepted for inclusion in Physics and Astronomy Faculty Research by an authorized administrator of Digital Commons @ Trinity. For more information, please contact jcostanz@trinity.edu. 


\title{
APPARATUS AND DEMONSTRATION NOTES
}

The downloaded PDF for any Note in this section contains all the Notes in this section.

John Essick, Editor

Department of Physics, Reed College, Portland, OR 97202

This department welcomes brief communications reporting new demonstrations, laboratory equipment, techniques, or materials of interest to teachers of physics. Notes on new applications of older apparatus, measurements supplementing data supplied by manufacturers, information which, while not new, is not generally known, procurement information, and news about apparatus under development may be suitable for publication in this section. Neither the American Journal of Physics nor the Editors assume responsibility for the correctness of the information presented.

Manuscripts should be submitted using the web-based system that can be accessed via the American Journal of Physics home page, http://web.mit.edu/rhprice/www, and will be forwarded to the ADN editor for consideration.

\section{LIGO analogy lab-A set of undergraduate lab experiments to demonstrate some principles of gravitational wave detection}

\author{
Dennis Ugolini and Hanna Rafferty ${ }^{a)}$ \\ Department of Physics and Astronomy, Trinity University, San Antonio, TX, USA 78232 \\ Max Winter, Carsten Rockstuhl, and Antje Bergmann ${ }^{\text {b) }}$ \\ Institut für Theoretische Festkörperphysik, Karlsruher Institut für Technologie (KIT), 76131 Karlsruhe, Germany
}

(Received 18 July 2018; accepted 10 October 2018)

\begin{abstract}
The first direct detection of gravitational waves by the Laser Interferometer Gravitational-Wave Observatory (LIGO) in September 2015 proved their existence, as predicted by Einstein's General Theory of Relativity, and ushered in the era of gravitational-wave interferometry. In this article, we present a set of lab course experiments at different levels of advancement, which give students insight into the basic LIGO operating principle and advanced detection techniques. Starting with methods for folding an optical cavity, we advance to analogy experiments with sound waves that can be detected with a Michelson interferometer with an optical cavity arm. In that experiment, students also learn how the sensitivity of the device can be tuned. In a last step, we show how optical heterodyne detection (the mixing of a signal with a reference oscillator) was used in Initial LIGO. We hope these experiments not only give students an understanding of some LIGO techniques but also awaken a fascination for how unimaginably tiny signals, created by powerful cosmic events a billion years ago or earlier, can be detected today here on Earth. ㄷ 2019 American Association of Physics Teachers.

https://doi.org/10.1119/1.5066567
\end{abstract}

\section{INTRODUCTION}

In September 2015, a sensational announcement excited the scientific community-the report of the first direct measurement of gravitational waves. The observed gravitationalwave signal was caused by the merger of a pair of black holes and was measured by the two detectors of the Laser Interferometer Gravitational-Wave Observatory (LIGO); the discovery was published in February $2016 .{ }^{1}$ The existence of gravitational waves had been postulated by Albert Einstein 100 years earlier ${ }^{2}$ but, before LIGO, the available instrumentation had not been advanced enough to directly measure these very weak signals. Now, with LIGO operational, more cosmic events can be detected; the latest observed signal was due to the coalescence of two neutron stars and was reported in October 2017. ${ }^{3}$ Given the new research frontiers opened up by LIGO, it was not surprising that the 2017 Nobel Prize in Physics was awarded to three physicists with leading roles in its development-Barry C. Barish, Kip S. Thorne, and Rainer Weiss ${ }^{4}$ - generating considerable interest in this topic among educators (and the public).
However, given the complexity of the LIGO experiment, it is a challenge to break down gravitational-wave and detector physics to an understandable level. Mathur et al. have suggested ideas on how to analyze LIGO data by using introductory physics, ${ }^{5}$ and Burko has published an introductory lab on the topic. ${ }^{6}$ Detailed information on the basic physics of the 2015 binary black hole merger (GW150914), which is suitable for educators and students, can be found in an article published by the LIGO Scientific and VIRGO collaborations. ${ }^{7}$ These articles suggest ways to analyze LIGO signals and are focused on theory. Since the fundamental functionality of LIGO is based on the combination of the well-known Michelson interferometer with optical cavity arms, in this paper we show that there is also the possibility of an experimental approach by means of educational analogy experiments.

The LIGO technology is highly intricate but features numerous experimental techniques and methods that can be extracted and studied individually. ${ }^{8}$ Some of these techniques can be understood by high school students, such as the Michelson Interferometer, while others are more suited to college students, like Pound-Drever-Hall laser frequency 
stabilization. ${ }^{9}$ In this paper, we present several undergraduate lab course activities that we have designed for different levels of expertise. The activities presented here contain basic interferometric techniques used in LIGO, an analogy experiment using sound waves symbolizing the gravitational waves, and an advanced experiment on optical cavity locking. Furthermore, we deliver components lists for readers who might be interested in setting up the experiments in their labs. Finally, we discuss the limits of the analogy experiments and the fundamental differences between gravitational waves and sound waves.

\section{DETECTING GRAVITATIONAL WAVES}

Gravitational waves are distortions of spacetime caused by the acceleration of massive astronomical bodies. They are predicted by Einstein's theory of general relativity to be transverse waves propagating at the speed of light. The waves should have quadrupole polarization, which means that if spacetime stretches in one direction, it will contract in the perpendicular direction. The amplitude of the waves is expressed as a strain $h=\frac{\Delta L}{L}$, that is, a change in length $\Delta L$ per unit length $L$. Because of the small size of the proportionality between stress and energy in Einstein's field equations $\left(\approx \frac{G}{c^{4}}\right)$, the strains of gravitational waves detected at Earth are on the order of $h \approx 10^{-21}$ or smaller. $^{6}$

A Michelson interferometer is ideal for measuring small changes in distance. This instrument is especially suited for detecting quadrupole radiation, since contracting one arm and extending the perpendicular arm strengthens the resulting signal. Given incident light of wavelength $\lambda$, a change in output from a bright fringe (constructive interference) to a dark fringe (destructive interference) requires a change of $\lambda / 4$ in the length of one arm compared to the other. The Advanced LIGO detectors have arm cavity lengths $L=4 \mathrm{~km}$ and use light from a solid-state Nd:YAG laser of wavelength $\lambda=1064 \mathrm{~nm},{ }^{10}$ so a change from a bright to a dark fringe represents a strain sensitivity given by

$$
h=\frac{\Delta L}{L}=\frac{\left(1.064 \times 10^{-6} \mathrm{~m}\right) / 4}{4 \times 10^{3} \mathrm{~m}} \approx 10^{-10} .
$$

Unfortunately, this value is eleven orders of magnitude larger than the required $h \approx 10^{-21}$ needed to resolve gravitational waves.

Further increasing the arm lengths quickly becomes unfeasible due to the curvature of Earth. But the sensitivity can be improved by folding the interferometer arms, or arranging the optics such that the light traverses the arms multiple times, effectively increasing their length. The original Michelson and Morley experiment used four mirrors at each end of an arm, with the light traveling in a zigzag pattern between them. ${ }^{11}$ Advanced LIGO adds a transmissive input mirror to each arm near the vertex, ${ }^{10}$ turning the arms into optical cavities with a finesse, i.e., average number of roundtrip reflections, of 450 . This innovation brings the strain sensitivity to nearly $10^{-13}$. The remaining eight orders of magnitude are achieved by "splitting" the fringe. Instead of simply determining if the output is "bright" or "dark," we measure how much the output changes from perfect destructive to perfect constructive interference, to better than one part in a billion. This is achieved through modulating the beam, which is explained below.
Figure 1 shows a simplified optical layout of the Advanced LIGO detector. The interferometer is operated near the dark fringe (the reason it is not exactly on the dark fringe is explained later in this section). This causes nearly all of the light returning to the beamsplitter to be dumped back towards the laser and wasted. More light in the interferometer is desirable to reduce shot noise, the fundamental quantum fluctuations in the number of photons in the beam, which decreases in significance as the laser power increases. A transmissive power recycling mirror (PRM) is added between the laser and beamsplitter to create another optical cavity, with the PRM at one end and the entire interferometer at the other. The cavity is resonant at the laser wavelength and reflects the light back into the interferometer. "Resonance" means that the length of the cavity is a multiple of half of the laser wavelength, such that each reflection in the cavity is in phase and constructively interferes with all others. Yet another transmissive mirror, the signal recycling mirror (SRM), is added at the output to enhance sensitivity at the frequencies where Advanced LIGO is most sensitive (about $100-300 \mathrm{~Hz}$ ). ${ }^{15}$ The SRM forms an optical cavity with the interferometer that is slightly detuned; it is resonant not for the laser frequency, but for beats between the laser and the expected gravitational-wave frequencies, such that these signals are preferentially extracted at the output. While essential to Advanced LIGO, these recycling cavities can be tricky to align, and are thus not included in our analogy experiments.

There are two potential issues with this arrangement. First, the detector must split a fringe to better than $10^{-9}$, while running with the output near the dark fringe. Second, positioning the arm cavity mirrors to within a tiny fraction of a wavelength requires precise sensing of whether the cavity is at resonance, but when resonant the cavity's transmission is at a maximum. In both cases, the optical system is operating at an extremum, where the slope is zero and the system is most insensitive to change.

To correct these issues, a linear signal is needed for precise measurement of changes in the interferometer arm lengths. This linear signal can be generated by modulating the laser (superimposing another frequency on the beam, either by amplitude modulation or phase modulation) and then mixing the interferometer output with the modulation frequency. Let the electric field of the modulated beam be described by

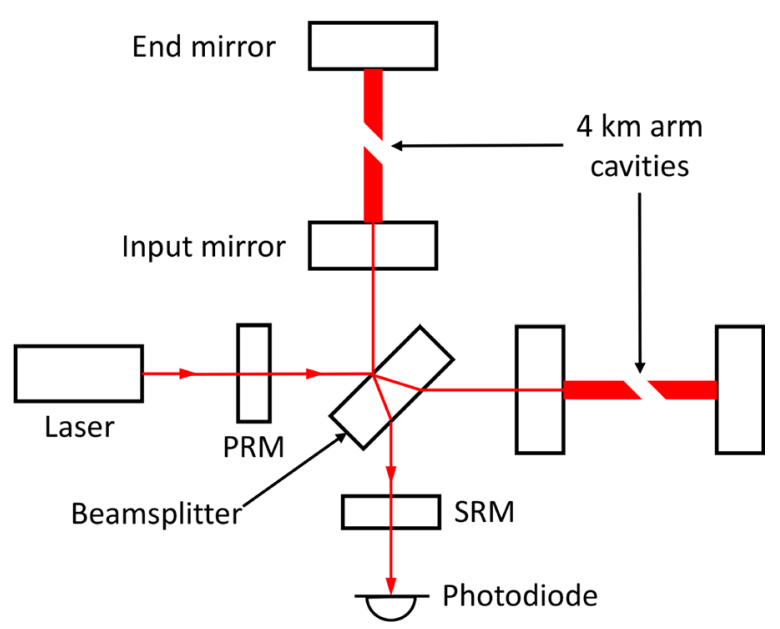

Fig. 1. Optical layout of the Advanced LIGO detector. 


$$
E=A \cos (\omega t)+B \cos \left(\omega+\omega_{m}\right) t+B \cos \left(\omega-\omega_{m}\right) t,
$$

where $A$ is the amplitude of the carrier (laser) wave, $B$ is the amplitude of the modulation sidebands (the beats between the carrier and modulation frequencies), $\omega$ is the carrier frequency, and $\omega_{m}$ is the modulation frequency. The carrier wave is resonant in the interferometer arms, while the modulation sidebands are not. At the output, a photodiode will measure the intensity of the wave. Thus we square the above expression and average over the carrier frequency to get

$$
I=\frac{1}{2} A^{2}+A B \cos \left(\omega_{m} t\right)+\frac{1}{2} B^{2} \cos \left(2 \omega_{m} t\right) .
$$

This signal is then mixed with the original modulation. The mechanism for mixing is described below, but the effect is that the signal is multiplied by a sine wave at the modulation frequency, yielding

$$
\begin{aligned}
I= & \frac{1}{2} A^{2} \cos \left(\omega_{m} t\right)+A B \cos ^{2}\left(\omega_{m} t\right) \\
& +\frac{1}{2} B^{2} \cos \left(\omega_{m} t\right) \cos \left(2 \omega_{m} t\right) .
\end{aligned}
$$

The mixed signal is then averaged over the modulation frequency. Only the term with amplitude $A B$ is nonzero, because cosine squared is always positive. And since $A$ is sensitive to deviations in the arm cavity from its resonant length and $B$ is not, the result is a linear error signal. Note that, using this method, gravitational waves are detected not by counting photons at the output, but by monitoring the linear error signal for the differential arm length. Initial LIGO used heterodyne detection, in which the output was mixed electronically with the modulation. To reduce electronic noise, Advanced LIGO uses homodyne detection, in which the carrier and sidebands mix optically at the output. In this approach, the interferometer must be moved slightly off the dark fringe; ${ }^{10}$ a little light must reach the output for optical mixing.

The following set of experiments are designed to highlight some of the most important enhancements beyond a basic Michelson interferometer in the Advanced LIGO detector. Students will learn how folding an interferometer arm will enhance sensitivity to length changes. They will measure sound waves as an analogy to the detection of gravitational waves. Finally, they will modulate the input beam and use heterodyne detection to generate a linear error signal, and use it to lock the optical cavity length on resonance.

\section{EDUCATIONAL ANALOGY EXPERIMENTS}

As described in Sec. II, the detection of gravitational waves is basically carried out using a Michelson interferometer (MI) with added transmissive mirrors in each arm to form optical cavities (OC). For our analogy experiments, we use a Michelson interferometer with only one arm turned into an optical cavity for easier alignment.

The setup is shown in Fig. 2, where (1) identifies a He-Ne laser, (2) the MI beamsplitter, and (3a) the input mirror of the optical cavity, which corresponds to the input test mass in the LIGO setup. (3b) labels the cavity's piezoelectricdriven output mirror, which is the end test mass in LIGO. (4) indicates the second MI mirror, which is moveable with a

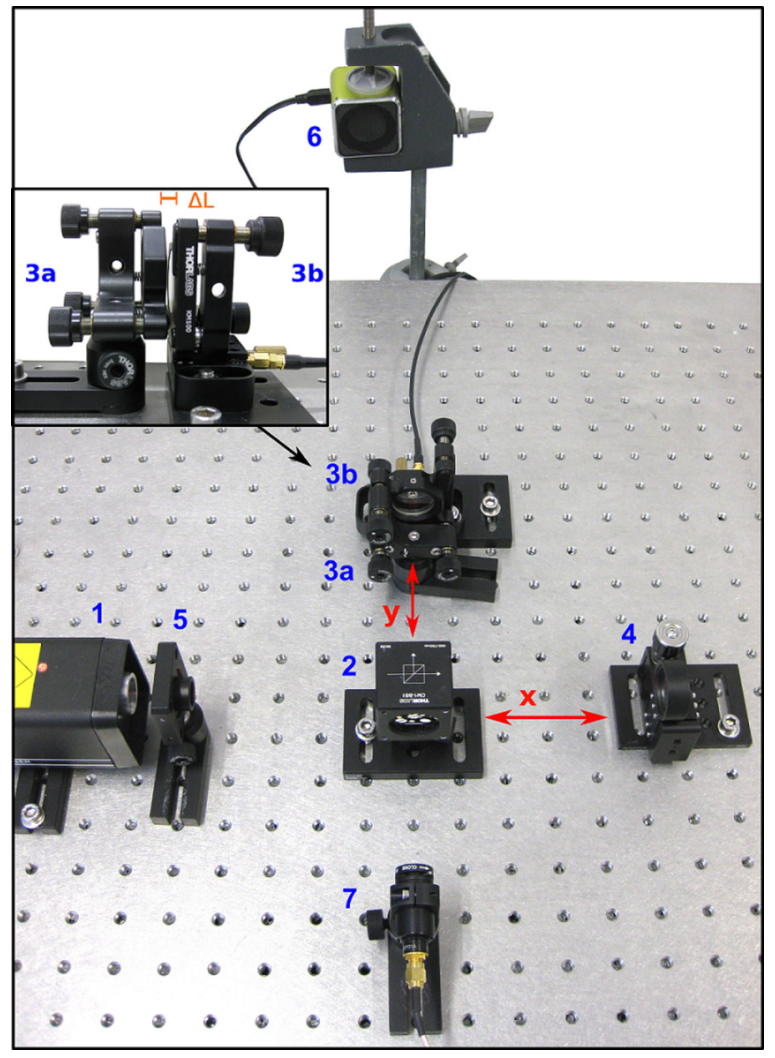

Fig. 2. Setup for the sound wave experiment: (1) He-Ne Laser, (2) MI beamsplitter, (3a) OC input mirror, (3b) OC output mirror, moveable with piezo, (4) second MI mirror, moveable with micrometer screw, (5) lens, (6) loudspeaker, (7) photodiode. Frame: close-up of the optical cavity with cavity length $\Delta L$.

micrometer screw, (5) a lens for diverging the laser beam and creating a fringe pattern, (6) the loudspeaker emitting the sound signal, and (7) the photodiode that is connected to a USB oscilloscope. In the following, we will call the MI arm without the optical cavity and the MI arm with the integrated cavity the $x$-arm and $y$-arm, respectively. The small frame shows a close-up of the optical cavity with the cavity length $\Delta L$. Details on the setup components are provided in the Appendix.

Figure 2 shows the basic setup used for all experiments. Each experiment entails slight changes to this setup, e.g., different beamsplitters are used for the experiments in Secs. III A and III B and further components are added in Sec. III C. Further descriptions are provided in the respective sections.

\section{A. Herriott delay line}

An optical cavity is not the only way to fold the interferometer arms. Another technique is called a Herriott delay line. ${ }^{12}$ Imagine a cavity formed by two spherical mirrors, one with an off-center hole drilled through it. A light beam parallel to the optical axis of the mirrors can be introduced through the hole in such a way that successive reflections "walk" around the perimeter of the mirrors until the beam reflects back out through the hole. The Herriott delay line is straightforward to design and align, but suffers from the fact that successive reflections must not overlap. Given the large arm length and number of reflections required for gravitational-wave astronomy, this results in impractical mirror sizes of a meter or more in diameter. ${ }^{13}$ 
As an analogy experiment, the delay line is an excellent way to demonstrate how each successive reflection in a folded arm increases the sensitivity to length changes. And while we cannot create a true delay line with our setup (since our mirrors are flat and have no drilled holes), we can use a misaligned cavity with a beamsplitter at one end to create several beams at the output, each representing a different number of round trips through the arm.

This experiment uses the basic setup in Fig. 2, where (2) is a 70:30 broadband plate beamsplitter and (3a) is a 50:50 broadband plate beamsplitter. The numbers before and after the colon are the percentage of light intensity reflected and transmitted, respectively; thus optic (2) reflects $70 \%$ of the light toward the delay line arm ( $y$-arm) and transmits $30 \%$ of the light to the $x$-arm. Optics (3a) and (3b) should be placed as close together as possible; the shorter the cavity, the easier it is to align. For this exercise, (6) and (7) will not be needed, and the interferometer output should be projected onto a distant screen.

Align the laser (1) onto the center of the Michelson beamsplitter (2), and ensure that the reflected and transmitted beams strike optics (3a) and (4), respectively. Next, align the delay line by steering ( $3 b)$ until it is parallel to (3a); when this is accomplished, all of the beams from the delay line should converge to a single spot at the output. Now slightly turn (3b) such that the delay line beams form a closely spaced line of spots at the output. The brightest represents a reflection off the front of (3a), while each successively dimmer spot represents one additional round trip through the arm. Make sure at least four spots are visible. Using a function generator, send a triangle wave to the piezo in (3b). The wave should be low in frequency $(0.02-0.1 \mathrm{~Hz})$ to allow enough time for counting fringes, and should have a positive DC offset such that the voltage never goes negative, since a reverse bias can damage the piezo.

Finally, align the beam from the $x$-arm (4) onto each output beam from the delay line in turn. Each round trip of the beam in the delay line should result in $X$ fringes per period of the triangle wave, where $X$ depends on the amplitude of the triangle wave. The first four spots should give zero, $X, 2 X$, and $3 X$ fringes per period, respectively. The brightest spot gives zero fringes because it is reflected off the front of the delay line, and is not sensitive to the delay line's length. Figure 3 shows the image of a row of beam spots that are multiple reflections from the delay line arm. Each dimmer spot represents one more round trip than the previous spot. The beam from the other arm is currently superimposed on the second-brightest spot, creating a fringe pattern.

\section{B. Experiments with sound waves}

\section{Setting up the Michelson interferometer with an optical cavity arm}

Section III A described experiments designed to teach students the physics of interferometers. In this section, we proceed to a next step and use the interferometer as an instrument for detecting sound waves. We demonstrate some basic aspects of the measurement of gravitational waves using a simple analogy experiment: A weak sinusoidal sound signal (representing the gravitational wave) is incident on the interferometer setup (representing the LIGO setup) and students are to extract the audio-produced sinusoid from the photodiode signal. The sound waves excite vibrations in the mirrors that cause very small changes in the lengths of the interferometer arms and produce phase shifts, i.e., light intensity variations in the interference pattern corresponding to the sound-wave frequency. These intensity variations, even if very small, can be detected with a photodiode. Furthermore, we use the combination of a Michelson interferometer and an optical cavity for these experiments, as it is done in the LIGO setup. We like to point out that the real LIGO experiment uses a much more sophisticated setup. Interested readers can find more information in the literature. $^{8,14-17}$ Before we start discussing the experiment, the interferometer needs to be aligned. Alignment is now a little different than in Sec. III A:

(1) Align the Michelson Interferometer (MI) so that the central maximum or minimum of the fringe pattern is as large as possible. This is the case if both arms of the interferometer are practically equal in length. Figure 4(a) shows how the respective interference pattern looks on a screen. For the moment, it is irrelevant whether there is a maximum or minimum in the central spot.

(2) Insert a beam splitter [= input mirror, (3a)] into the interferometer arm ( $y$-arm) with the piezo-driven output mirror (3b) to form an optical cavity (OC) [see Fig. 2, ( $3 \mathrm{a}, 3 \mathrm{~b})$; a close-up is shown in the small frame] as shown in Sec. III A. Align the OC so that the fringe pattern is concentric to the MI fringe pattern. When aligning the OC you can just cover the second MI mirror (4) with a piece of paper. Figure 4(b) shows how the OC fringes look without the MI.

(3) Take the piece of paper out of the $x$-arm. You will see both interference patterns overlapping on the screen. Use the alignment screws on mirror ( $3 \mathrm{a}$ ) to superimpose both fringe patterns concentrically. If both the MI and the OC are aligned correctly, the pattern is supposed to look as

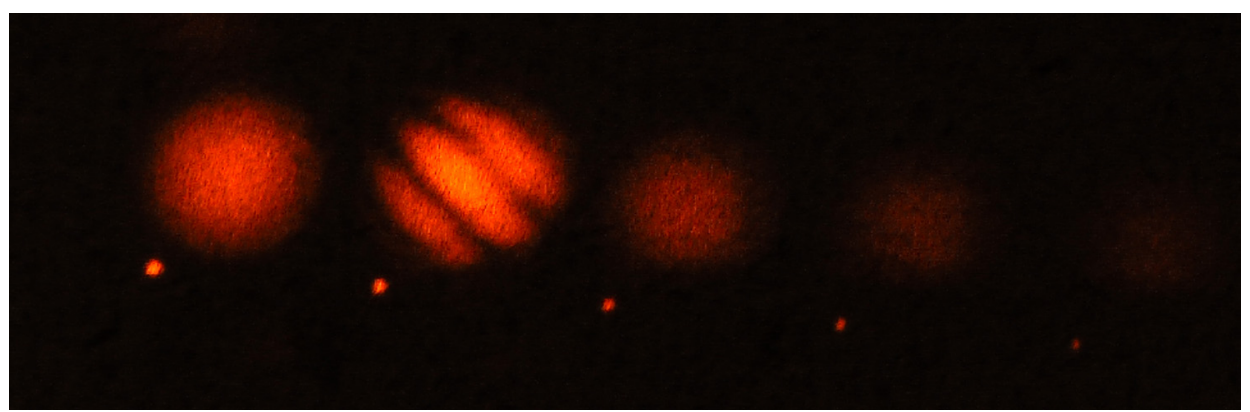

Fig. 3. Reflections from the delay line arm ( $y$-arm). The second-brightest spot shows interference created by superposition of light from the $x$ - and $y$-arm. 

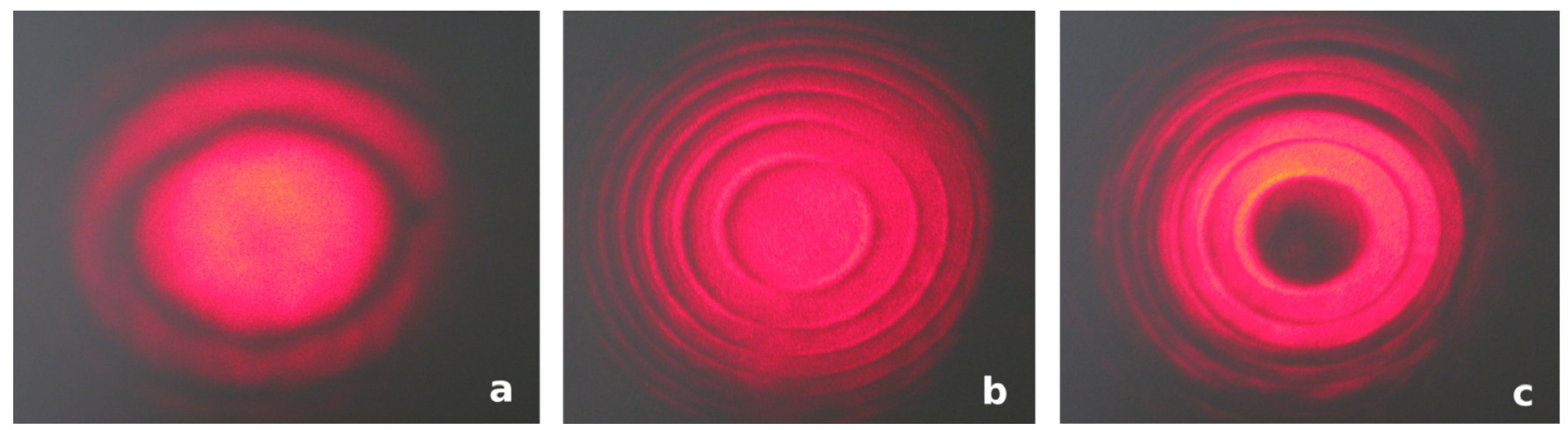

Fig. 4. Interference fringes: (a) Michelson interferometer, (b) optical cavity, (c) MI with OC arm.

in Fig. 4(c). For now it does not matter whether there is an intensity minimum or maximum in the center.

(4) Put a loudspeaker [simple PC speaker will do, as (6)] close to the setup. Make sure not to attach the speaker to the interferometer breadboard. Mechanical vibrations from the speaker might be transmitted to the mirror directly via the breadboard. Obviously, this is undesirable for didactical reasons.

(5) Put the photodiode (7) in the center of the combined fringe pattern.

Before we start the experiments, we discuss some properties of the Michelson interferometer with an optical cavity arm.

\section{Sensitivity of the Michelson interferometer with an optical cavity arm}

For measuring weak signals, we want our setup to have the highest possible sensitivity, which can be achieved by adjusting the operating point of our interferometer system. We discuss how to adjust the operating point in the following.

The output intensity of the MI + OC system depends on the vector sum of the complex amplitudes of both arms, containing real amplitudes and phases. A full discussion of this concept goes far beyond the scope of this article, so we encourage the interested reader to study the literature. We recommend an article by Black and Gutenkunst, ${ }^{8}$ which provides a very thorough and helpful discussion on this topic. In brief, we will only use the very basic ideas for our explanations: In our setup, we can change the contribution of the complex amplitude of the $x$-arm by adjusting the arm length with the micrometer screw of mirror (4). In addition, the rate of change of the phase of the reflected light in response to a change in arm length is greatest, i.e., the interferometer is most sensitive, when the arm cavity is in resonance. That is, in resonance, the interferometer is most sensitive to changes in the distance between the mirrors (which is favorable), since small mirror deviations result in high intensity variations measured by our photodiode.

By independently adjusting the arm lengths of both interferometers, we can tune the sensitivity of our instrument. Above, we have called this process adjusting the "operating point." To give the students an idea how to achieve the optimal operating point, we let them carry out a preliminary study in which they can observe various situations as follows: We oscillate the optical cavity by sending a triangle wave from the function generator to the output mirror piezo
[Fig. 2, (3b)], and detect the output signal with a photodiode (7) connected to an oscilloscope. By doing so, we obtain a linear translation of the mirror for a certain time period, which corresponds to a linear change in cavity length. During that period, we can observe several transitions through the cavity resonance. Furthermore, we can adjust the length of the $x$-arm with the micrometer screw on mirror (4).

Figure 5 shows screen shots of two exemplary situations recorded from our $\mathrm{MI}+\mathrm{OC}$ setup with an optical cavity consisting of a $50 \%$ reflecting input and $100 \%$ reflecting output mirror. The two situations represent two different contributions of the $x$-arm to the system, practically meaning two differently adjusted lengths of the $x$-arm. Channel 1 , the upper trace, shows the photodiode signal, while Channel 2, the lower trace, represents the input signal of the function generator, which only shows a linearly increasing section of the triangle voltage. The higher the voltage ( $y$-axis), the higher the measured light intensity at the photodiode (absolute value is irrelevant). The drawn dots mark the resonance positions of the optical cavity length. It can be seen that, e.g., if we adjust our system so that our operating point is located on resonance in the situation shown in Fig. 5(a) we obtain a high intensity gradient with respect to length changes of the cavity, i.e., a high sensitivity. In the situation Fig. 5(b) there is a reversal point and an intensity minimum at resonance. Thus, it is favorable to align the interferometers as in Fig. 5(a) for measuring with high sensitivity. In this way, very weak incoming sound signals with very small amplitude oscillations of our cavity mirrors result in relatively large light intensity variations at our photodiode, providing a larger signal. Again, it should be noted that the choice of the operating point for our sound experiment is not the same as in the LIGO experiment as described in Sec. II.

After we have found how the Michelson interferometer with an optical cavity needs to be tuned to obtain the highest possible sensitivity, it is also interesting to compare this system's sensitivity with a Michelson interferometer without a cavity. To analyze this, we have excited both systems (MI alone and $\mathrm{MI}+\mathrm{OC}$ tuned on resonance) with a $670 \mathrm{~Hz}$ signal via the piezo at the end mirrors (3b) in the $y$-arm, simulating a stable incoming signal. It should be noted that we used the same piezo for excitation as we did for tuning the optical cavity in the $y$-arm. However, for the excitation we applied very small piezo amplitudes on the order of fractions of the laser wavelength, so that the resulting oscillations are only very small deviations from resonance in the cavity (and not actually detuning the cavity). Of course, we have been careful to use exactly the same parameters (such as the MI arm 


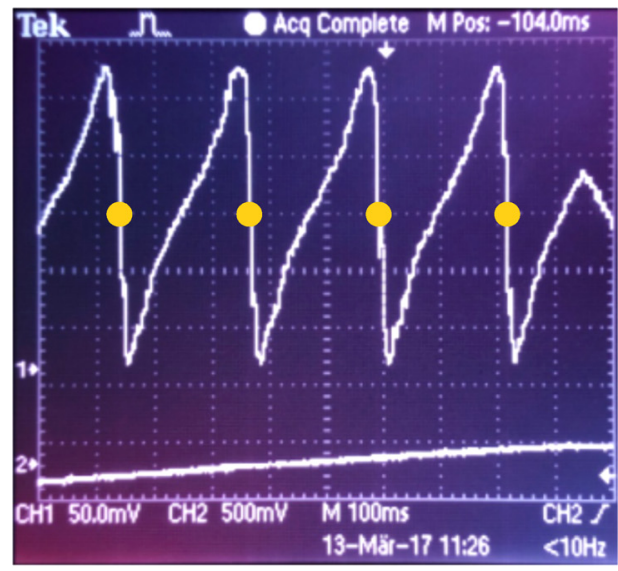

a)

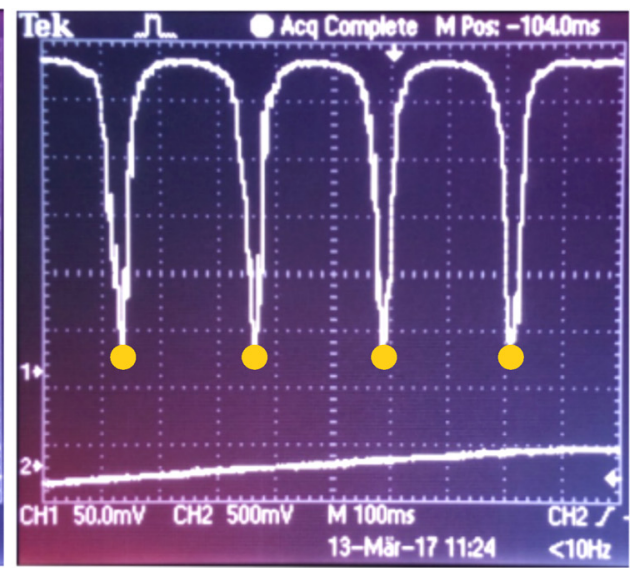

b)

Fig. 5. Output signals for two situations, i.e., two different lengths of the $x$-arm. The drawn dots mark the resonance positions of the optical cavity in the $y$ arm. The full mathematical derivation of these waveforms can be calculated using the model presented in Black and Gutenkunst(Ref. 8), Section VI: (a) corresponds to a constant contribution of $-\frac{1}{2} \mathrm{e}^{-i \frac{\pi}{2}}$ of the $x$-arm to the complex amplitude, (b) to a contribution of $-\frac{1}{2}$.

lengths, excitation amplitude, and so on) for both systems. Then, we have measured the signal amplitude with the photodiode for each setup. We carried out 45 measurements for each system; Table I displays the obtained mean values.

The results in Table I show that a much higher signal amplitude, $-45.9 \mathrm{dBu}$, can be achieved by inserting an optical cavity into a Michelson interferometer arm. Without a cavity, the signal amplitude of the MI only yields $-62.1 \mathrm{dBu}$ (note that $\mathrm{dBu}$ units are logarithmic). We have to mentioned that the optical cavity requires careful and precise tuning (i.e., finding the correct operating point as mentioned above) to see the effect clearly. Unexperienced students might have a little trouble achieving such clear results. On the other hand, the students will learn from this experience that accurate tuning is essential, both for LIGO and the analogy experiments.

\section{Detecting weak sound signals}

After the preliminary studies, the sound wave experiments are carried out. First of all, the interferometers need to be adjusted to the operating point, then the sound signal can be sent:

(1) Tune the interferometer setup: Move the OC output mirror (3b) with the piezo using a triangle voltage from the function generator, i.e., change the cavity length continuously. Observe the photodiode signal with the oscilloscope.

(2) Adjust the $x$-arm using the micrometer screw on mirror (4) until the situation as shown in Fig. 5(a) is evident. Note the voltage at the resonance points (drawn dots). Switch off the function generator and move the piezo to the resonance position of the cavity, as noted.

(3) Now, observe the photodiode signal with an oscilloscope using the FFT representation (frequency spectrum) without an incoming sound signal. Record 30-50 samples

Table I. Average amplitude of photodiode signal at $670 \mathrm{~Hz}$.

\begin{tabular}{lcc}
\hline \hline Amplitude & MI & MI + OC \\
\hline Mean value, 45 measurements & $-62.1 \mathrm{dBu}$ & $-45.9 \mathrm{dBu}$ \\
\hline \hline
\end{tabular}

and average them (using the averaging function of the oscilloscope software).

(4) Next, send a sine-wave sound via the loudspeaker and observe the photodiode signal as in the previous step.

Figure 6 shows the frequency spectrum from 0 to $2 \mathrm{kHz}$ of the photodiode signal without a sound signal. The $y$-axis shows the voltage level in $\mathrm{dBu}$ units. We observe several intensity peaks in the frequency range below approximately $0.25 \mathrm{kHz}$. Since these data were taken with no incident sound signal, these peaks are clearly background noise caused by, e.g., vibrations like seismic noise and activities inside or outside the building. Therefore, it obviously is problematic to measure signals in this frequency range. It should be mentioned that this background noise will be different depending on the building in which the setup is used or on the day when measurements are carried out (e.g., one day, there were construction crews close to the building producing large unwanted signals). This noise also depends on the components used (e.g., breadboard, mounting equipment). This noise problem is an interesting topic to discuss in the lab course since it is of utmost importance in gravitational wave detection experiments, where numerous different sources of noise need to be considered and reduced, including anthropogenic noise, earthquakes, ${ }^{18}$ and quantum noise. ${ }^{16,19}$

When sending a very weak sound signal (in order to mimic a gravitational wave) into the setup, it is advisable to choose a frequency above approximately $0.25 \mathrm{kHz}$ to avoid the high-intensity noise region. We used $650 \mathrm{~Hz}$ and turned down the volume of the loudspeaker until the sound was barely audible. In Fig. 7, the recorded frequency spectrum can be seen, again averaged over 50 samples. The $650 \mathrm{~Hz}$ signal is observed as a single peak in the spectrum, standing out clearly from the background noise. A second peak at $1.3 \mathrm{kHz}$ is most likely due to a second harmonic excitation. Note that, in our experiment, we have plenty of time (several seconds) to record the signal of our continuous sound wave, while LIGO must reliably capture the signals from cosmic events that last only fractions of a second.

We can now study the sensitivity of our setup by using the piezo to change the cavity length by $\Delta L$ and tune the system a little bit off the resonance point. We carry out measurements at different positions, on and off resonance, and 


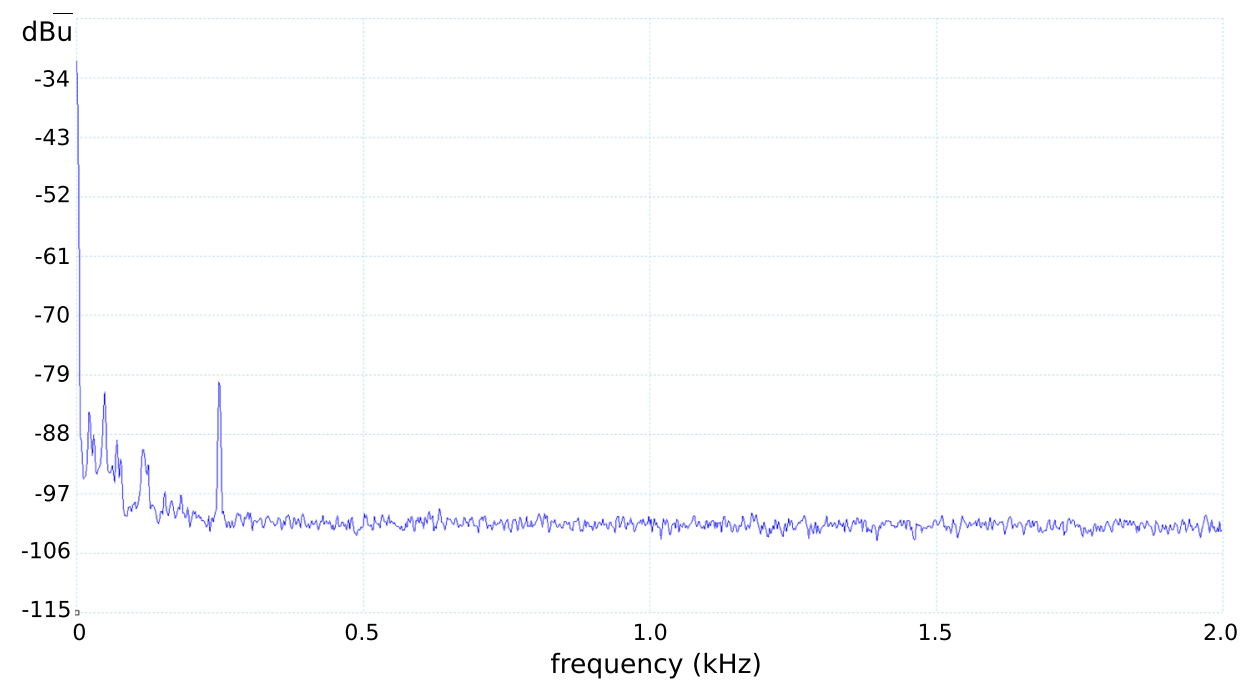

Fig. 6. Frequency spectrum of the background noise between 0 and approximately $2 \mathrm{kHz}$.

observe both the noise and the signal level. Figure 8 shows another two frequency spectra from 0 to $1 \mathrm{kHz}$ with a $650 \mathrm{~Hz}$ sound signal recorded. Figures 8(a) and 8(b) show a properly tuned (on-resonance) optical cavity and a detuned (off-resonance) optical cavity, respectively. Each spectrum was obtained by recording and averaging 50 samples, which took about $15 \mathrm{~s}$; the volume level of the incoming sound wave was equal for both measurements. The arrows mark the $650 \mathrm{~Hz}$ sound signals. It is obvious that the well-tuned cavity [Fig. 8(a)] provides a clear signal with a voltage level of $-64 \mathrm{dBu}$. With the badly tuned cavity [Fig. 8(b)], only a very weak signal can be surmised at $650 \mathrm{~Hz}$, the voltage level being at approximately $-82 \mathrm{dBu}$. It is also noticeable that the noise level in Fig. 8(a) is much higher (up to -64 $\mathrm{dBu}$ ) than in Fig. 8(b) (up to approximately $-80 \mathrm{dBu}$ ).

So it becomes clear that the detection sensitivity of the well-tuned cavity is much higher than in the off-resonance case. Both the signal and the noise rise to higher levels in the well-tuned case, so an increase in sensitivity comes at the expense of a higher noise level. In order to deal with the noise we use two strategies:
- We can try to influence the mostly seismic noise background by changing our setup parameters. We can, for example, choose specific mirrors, breadboards, tables, etc., to shift the noise to frequencies at which we do not expect signals. We tried several breadboards and optical components until we were satisfied with our result. Finally, as can be seen in all the presented figures, we have to deal with noise mostly below $250 \mathrm{~Hz}$. Above that there is a large frequency range with relatively low noise that works well for us. While we can try to reduce that noise by simply using suitable standard breadboards and tables, LIGO needs to put tremendous effort into their seismic noise attenuation system. $^{20}$

- We mostly deal with noise that does not come from one single source, but has a statistically random component, e.g., people walking around in the building, cars passing by on the streets next to the building. Thus, we are able to better distinguish between noise and the wanted signal by averaging several measurements, provided that the signal level exceeds the noise level at a given frequency. We usually took between 10 and 50 samples to obtain really

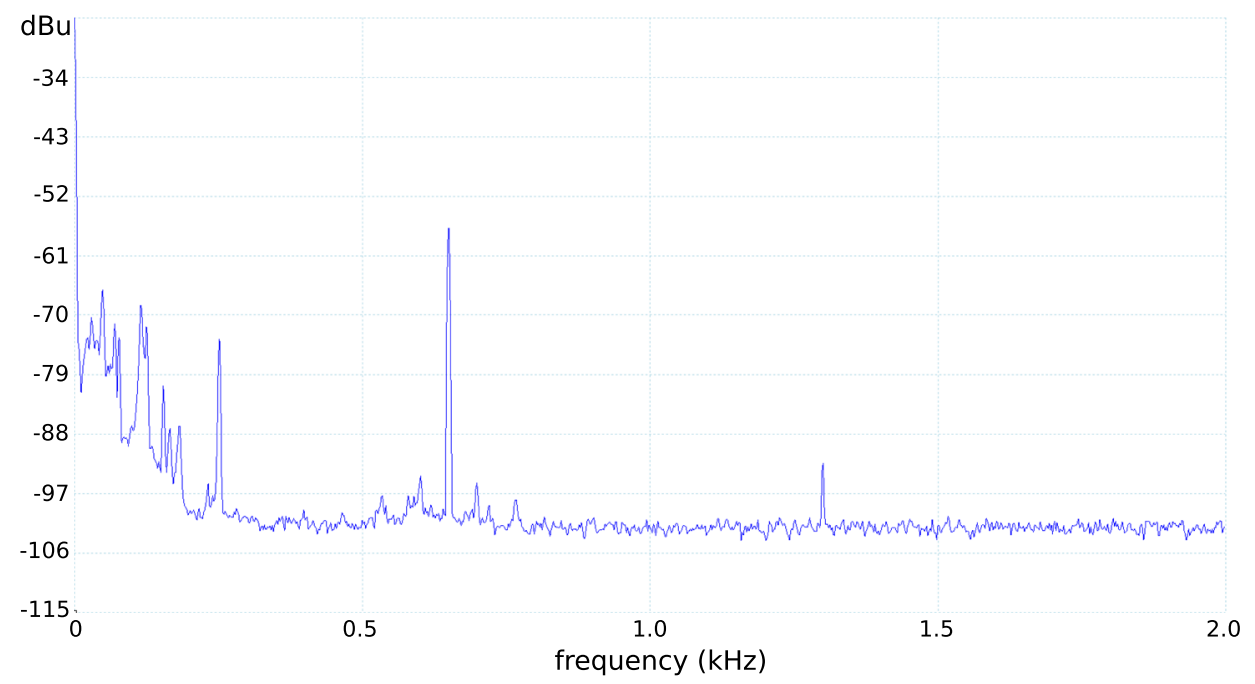

Fig. 7. Frequency spectrum with a $650 \mathrm{~Hz}$ sound signal input. 


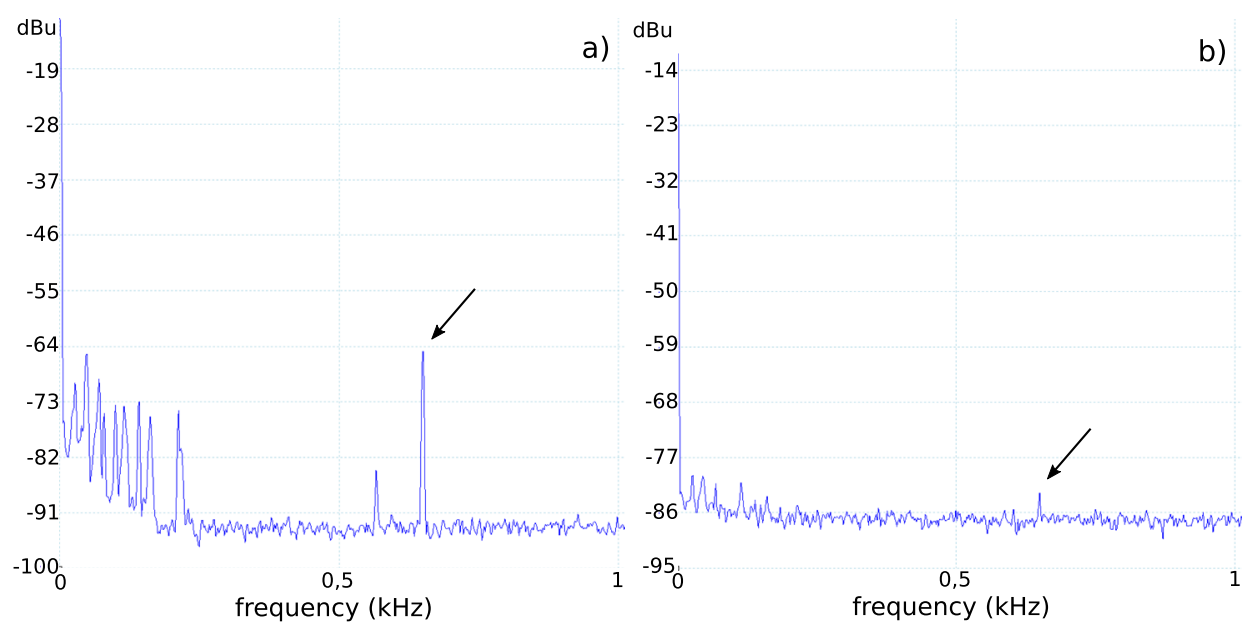

Fig. 8. Frequency spectrum with a $650 \mathrm{~Hz}$ sound signal: (a) cavity tuned on resonance, (b) cavity off resonance.

good results. If the signal is strong enough, one sample is enough to detect it, however, the signal-to-noise ratio will of course be higher than when averaging several samples.

Another important question concerning the setup sensitivity is its frequency dependency. Before analyzing that we need to consider the frequency response of the loudspeakers being used, i.e., the frequency dependency of the emitted sound intensity. Since we used low-cost speakers, no response curve was provided by the manufacturer, so we measured it with a calibrated sound level meter. We found that the response characteristic is relatively flat in the frequency range that we used for our signal analysis, between $400 \mathrm{~Hz}$ and $2 \mathrm{kHz}$. Variations were about $\pm 1.5 \mathrm{~dB}$. Below $400 \mathrm{~Hz}$ the response decreased strongly, so we did not use this frequency range for our signal analysis.

Every system subjected to vibrations has a self-resonance at specific frequencies and therefore the system sensitivity is also frequency-dependent. Thus we analyzed the frequency dependence of our setup by exposing it to a sound frequency sweep from $0.4 \mathrm{kHz}$ to $2 \mathrm{kHz}$ (over a time span of $4 \mathrm{~s}$ ) in discrete steps of $100 \mathrm{~Hz}$. One must be aware that by using only discrete narrow-bandwidth frequencies we miss many frequencies/resonances in between. However, performing a more finely resolved sweep can become very time-consuming. Therefore, we did not record a full frequency-response curve, but instead only investigated quantitatively the general frequency dependence.

During the sweep the volume level was constant, as mentioned above. For the signal acquisition we used the oscilloscope software function for "peak amplitude recording." With this function, the first spectrum is recorded and the data is saved temporarily. Then, the next measurement is taken and all amplitudes in this spectrum are compared with those of the first measurement. The highest values are adopted and saved. Then, the third measurement is taken, comparing the values with the previously saved ones, and so on. This process is done during the whole signal sweep; the result can be seen in Fig. 9. The differences in sensitivity can therefore be identified by comparing the amplitudes of the frequencies at which the sound signals were applied.

The highest signal amplitudes can be observed between $400 \mathrm{~Hz}$ and $700 \mathrm{~Hz}$. Between $800 \mathrm{~Hz}$ and $1.3 \mathrm{kHz}$, the signal is still clearly observable but the amplitude is lower by about $20 \mathrm{dBu}$ when comparing the highest signal levels in these

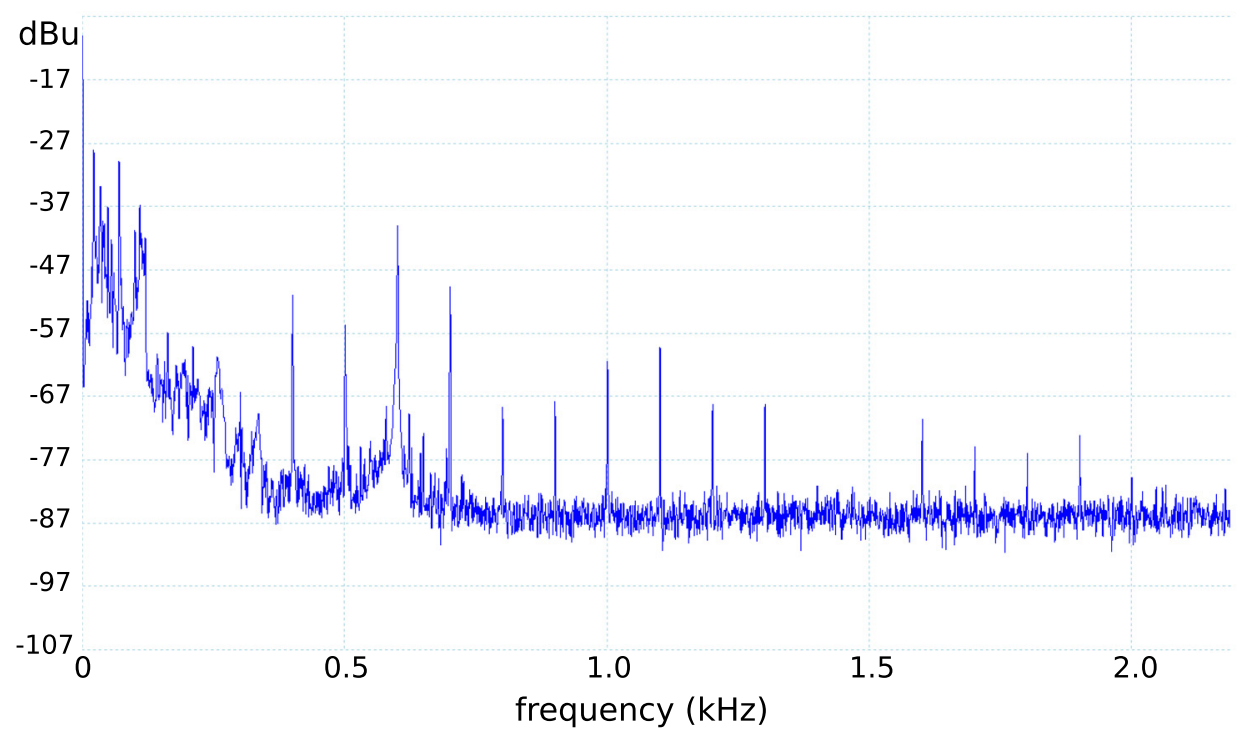

Fig. 9. Recorded sound signals in steps of $100 \mathrm{~Hz}$ captured by peak amplitude recording. 
two frequency regions. Above $1.4 \mathrm{kHz}$ a significant decrease in signal intensity can be observed. At $1.4,1.5$, and $2 \mathrm{kHz}$ the signal can hardly be detected. It is evident that there is a strong frequency dependence. Due to the relatively flat response curve of the loudspeakers, as considered above, we can be sure that this is a characteristic of our setup. It should be mentioned that we did not consider using even higher frequencies simply due to the fact that frequencies above $2 \mathrm{kHz}$ are extremely unpleasant to listen to while experimenting. Finally, we note that sound signals can be detected well with our setup in an adequate frequency range useable for lab experiments. However, the signal intensity strongly depends on the signal frequency. This is also true for the real experiment: LIGO must carefully calibrate, so that for every part of the sensitive frequency range, it is known what measured signal size corresponds to what gravitational-wave strain. The LIGO detectors' sensitivity extends from $10 \mathrm{~Hz}$ to $10 \mathrm{kHz}{ }^{21}$

The LIGO Open Science Center provides actual chirp sounds for free download. ${ }^{22}$ Audio files from various events can be chosen and used as a sound signal as described above.

\section{Advanced experiments: Locking the optical cavity to resonance}

In our last experiment, we will lock an optical cavity by using heterodyne detection to generate a linear error signal. First we must modulate the laser beam. In LIGO, the light is phase modulated by applying a sinusoidal voltage to an electro-optic modulator (EOM). Since an EOM can cost more than the rest of our setup combined, we will instead phase modulate the light via the Doppler effect by aligning the beam at near-normal incidence on a PZT-mounted mirror (PZT: Lead Zirconate Titanate piezo ceramic), and driving the PZT with a sinusoidal voltage.

The optical layout is shown in Fig. 10. The laser (1) is aligned through a converging lens (2) into a triangular path of mirrors (3). The PZT-mounted modulating mirror (4) is at the apex of this isosceles triangle. For best results, we

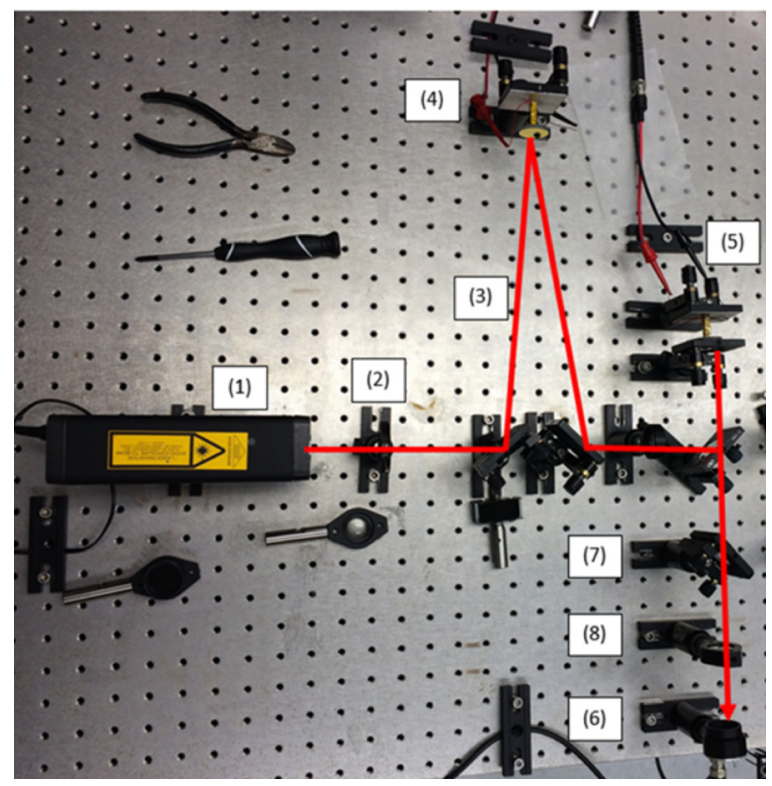

Fig. 10. Optical layout for locking optical cavity, including (1) laser, (2) focusing lens, (3) triangular path of mirrors, (4) PZT-driven modulation mirror, (5) optical cavity, (6) photodiode, (7) pickoff beamsplitter, and (8) diverging lens or neutral density filter. minimize the base and maximize the height of the triangle (within the limits of how far the beam will stay focused) so that the beam arrives as close to normal incidence as possible, and align the beam onto the center of the PZT-driven mirror. The beam then reflects off a beamsplitter into an optical cavity (5). Note that we do not need the other interferometer arm for this experiment, so the transmitted beam can be blocked. The reflected beam from the cavity is aligned onto a photodiode (6). A pickoff beamsplitter (7) can be used to confirm alignment of the cavity, and if the beam is too bright for the photodiode, a diverging lens or neutral density filter (8) can reduce the intensity. The modulation mirror (4) should be driven with a large-amplitude sine wave at the resonance frequency of the PZT. We had good results with a Thorlabs Discrete Piezo Stack (Model PK2FQP2, $19.6 \mu \mathrm{m}$ displacement at $75 \mathrm{~V}, 65 \mathrm{kHz}$ resonance) driven with a $5 \mathrm{~V}$ peak-to-peak sine wave, yielding approximately $1.3 \mu \mathrm{m}$ of motion. The cavity mirror (5) should be swept with a low frequency $(\approx 1 \mathrm{~Hz})$ triangle wave such that the cavity passes at least from one bright fringe to the next.

The mixer circuit for generating a linear error signal is shown in Fig. 11. The same sinusoidal voltage sent to the modulation mirror is also applied to "Modulation Sync Input" of the mixer circuit, where it goes through a high-pass filter to eliminate any DC offset. It then enters a phase shifter; adjusting potentiometer R5 will sweep the signal through $180^{\circ}$ of phase change, to allow the user to find the phase that gives the maximum signal from the mixer. The photodiode signal is applied to a bandpass filter to remove DC offset and high-frequency noise. Both of these signals are then mixed in an Analog Devices AD630 modulator/ demodulator IC arranged as a lock-in amplifier. ${ }^{23}$ A simple multiplier will also work, but we found far better results with the AD630 at trivially higher cost. The AD630 output passes through a $100 \times$ amplifier and two-pole $160 \mathrm{~Hz}$ filter that acts as an integrator over the modulation frequency.

Once the optical layout and mixer circuit are completed and the optical cavity is aligned, use the cavity PZT driving signal to trigger an oscilloscope (see the blue trace in Fig. 12) and observe at "Scope Output" from the mixer circuit. With some tuning of the phase shifter, the "Scope Output" should match the yellow trace in Fig. 12. The center of the linear error signal, where it crosses zero voltage with positive slope in Fig. 12, is the point at which the optical cavity is at resonance. If your cavity sweep is sufficiently large, you may see more than one resonant mode per sweep. If you are unable to generate this oscilloscope image, the most likely culprit is that the modulation is too weak; either the beam is not centered on the PZT-driven mirror, the driving signal amplitude is too small, or the driving signal is not at the PZT resonance frequency.

Now we are ready to lock the optical cavity at resonance. Figure 13 shows the feedback circuit for accomplishing the resonance lock. The linear error signal from "Scope Output" in the mixing circuit is applied to "Mixer Output" in the feedback circuit of Fig. 13, where the error signal is added to a voltage offset controlled by potentiometer R3. This allows the user to put the resonant condition (the center of the linear error signal) at zero voltage, such that the sign of the error signal determines the direction of feedback to the cavity PZT. The signal then passes through an amplifier with adjustable gain controlled by potentiometer R6, followed by an inverting/non-inverting amplifier controlled by a polarity switch. This is not only good practice for feedback circuits, 


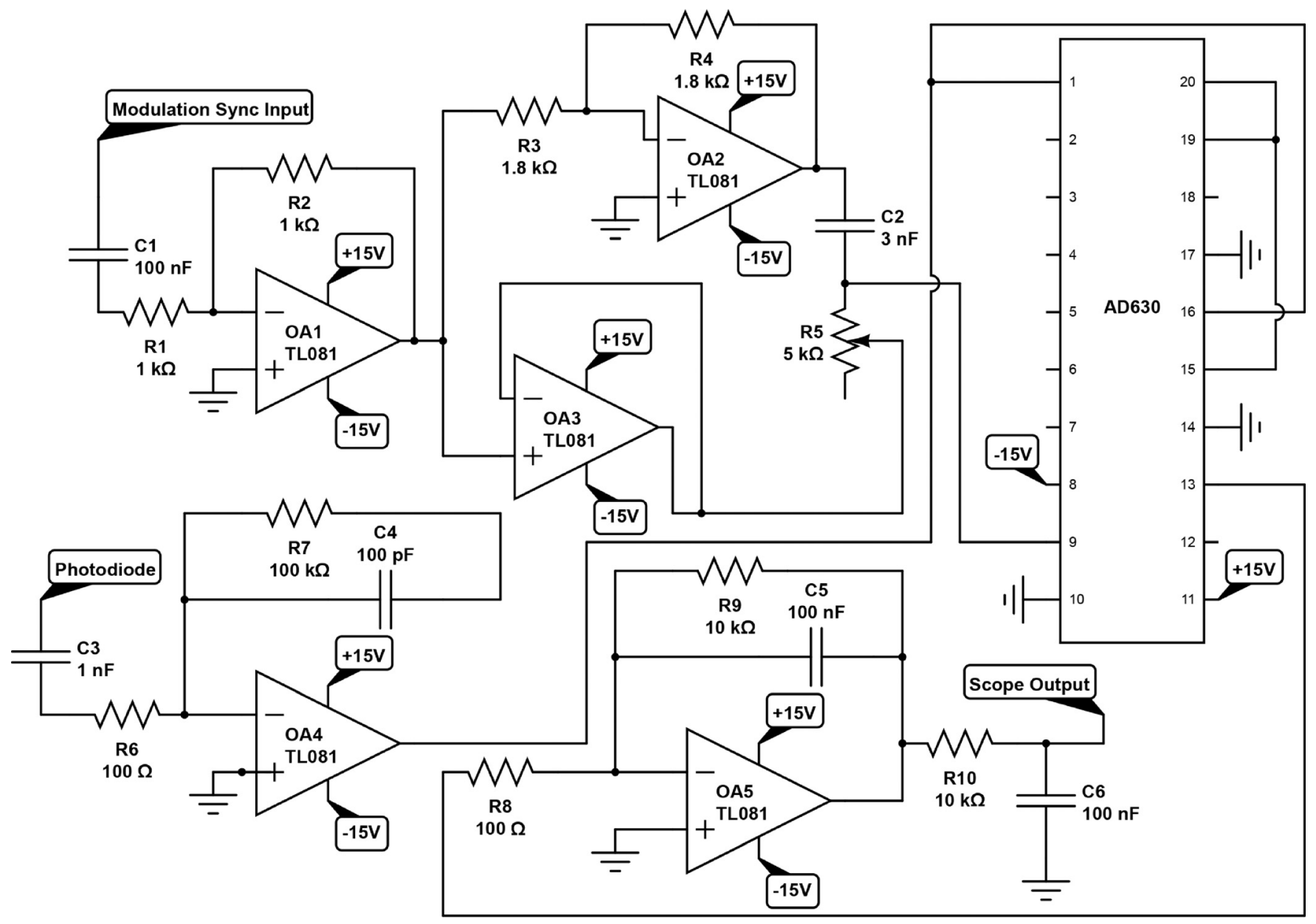

Fig. 11. Mixing circuit for generating linear error signal by heterodyning photodiode output and modulation frequency.

but will allow students to see how positive feedback induces oscillations. The feedback signal can be observed at "Sig Mon," and an on/off switch controls whether it is sent to the PZT. The feedback signal is added to a triangle wave (applied at "Ramp In") and a voltage adjusted with potentiometer "Ramp Offset." This design keeps the resonant condition centered both in the cavity ramp and on the

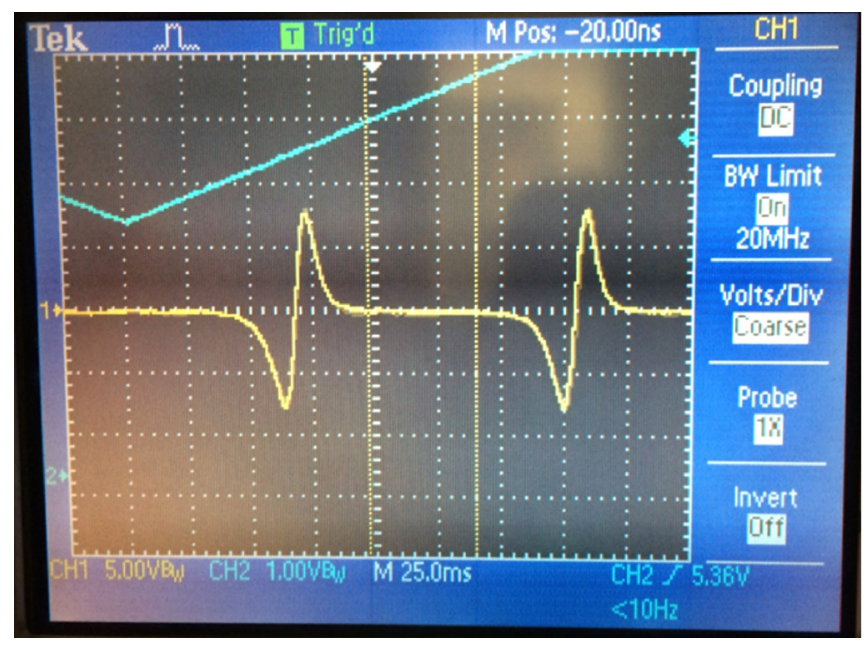

Fig. 12. Output of mixer circuit. The top trace is the cavity PZT ramp while the bottom trace is the mixer output. There are two error signals in the output, representing two different resonant modes of the cavity. oscilloscope screen. Finally, a low-pass filter creates a unitygain frequency around $500 \mathrm{~Hz}$ to avoid positive feedback at higher frequencies, and the signal is sent to the cavity PZT.

To lock the cavity, use the triangle wave to trigger the oscilloscope, set the time base so that one-half period of the triangle wave fills the screen, and observe the mixer output. Use R3 to center the error signal vertically at zero volts, and use Ramp Offset to center the error signal horizontally. Keep the gain low and the feedback on/off switch in the off position. Gradually reduce the amplitude of the triangle wave, which will widen the error signal on the oscilloscope screen. Continually use R3 and Ramp Offset to re-center the signal vertically and horizontally. When only the linear portion of the error signal fills the whole oscilloscope screen, switch on the feedback. If the signal looks like the left image in Fig. 14, you are applying positive feedback; flip the polarity switch and repeat the locking procedure. If the signal looks like the right image in Fig. 14, the cavity is locked to resonance. Shut off the cavity PZT ramp and turn up the feedback gain. The beam from the pickoff beamsplitter should be stable, with no visible change in intensity.

\section{Educational notes}

Sound waves acting on a Michelson interferometer with an optical cavity arm is a comprehensible analogy to LIGO and can help students understand some basic principles of the detection of gravitational waves. Both sound and 


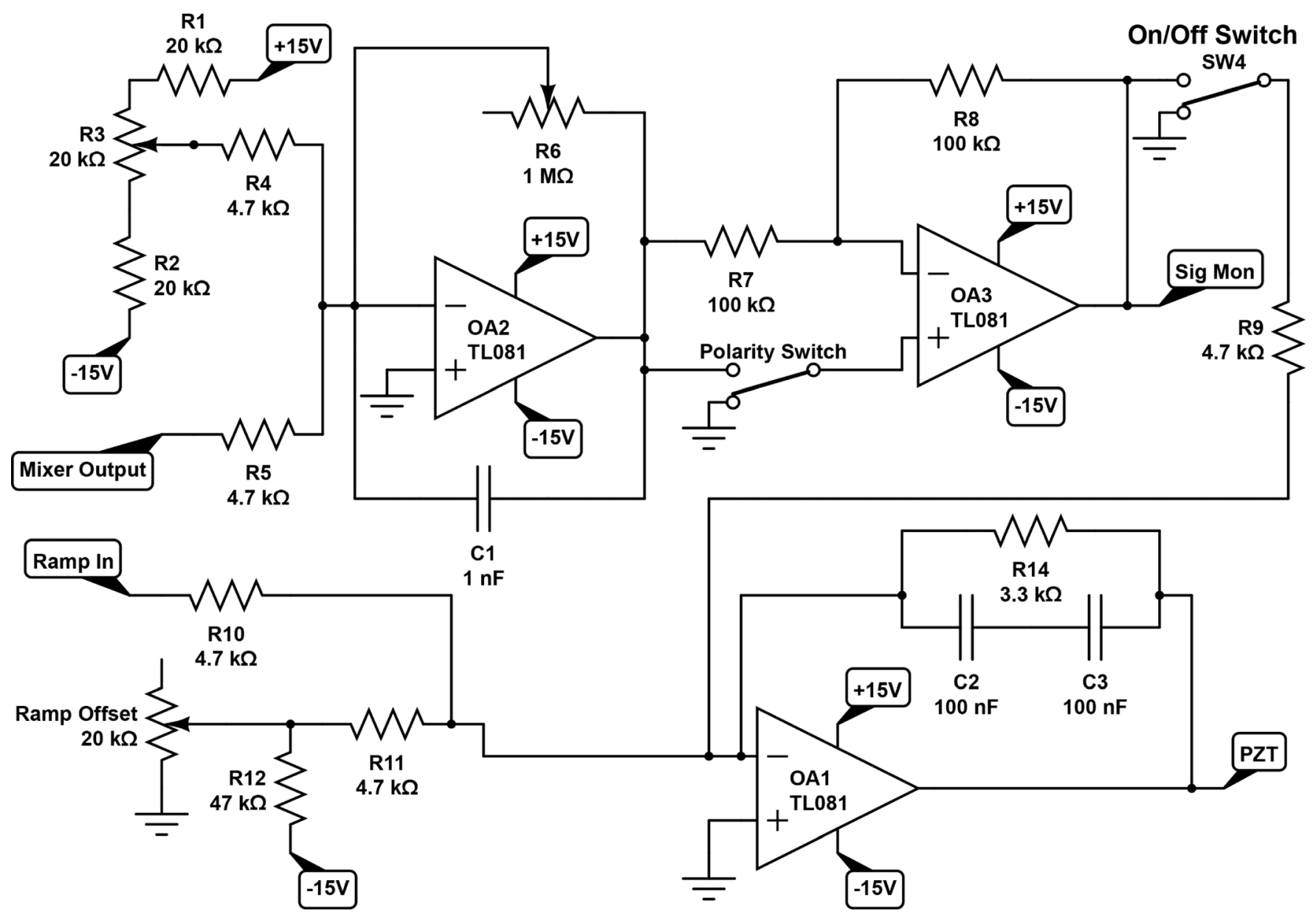

Fig. 13. Feedback circuit for locking the optical cavity. The linear error signal from the mixing circuit in Fig. 11 is applied at "Mixer Output," and the feedback signal to control the optical cavity length is output at "PZT."

gravitational waves are sinusoidal waves and their interaction with the interferometer influences the lengths of the Michelson Interferometer arms and the optical cavity, causing intensity variations at the detector.

However, there are fundamental differences that need to be discussed when performing this kind of analogy experiment. Listed here are the most important issues (for theory details see Sec. II):

- Gravitational waves cause perturbations in spacetime. Sound waves are waves of pressure propagating through a medium (gas, liquid, or solid).
- Gravitational waves are transverse waves propagating with the speed of light; sound waves are longitudinal waves propagating with the speed of sound.

- Gravitational waves act on spacetime, stretching in one axis and squeezing in the other axis and the other way round half a period later. Thus, in a Michelson Interferometer, one arm is shortened, while the other is lengthened. Sound waves influence the interferometer by exciting vibrations in its components (mirrors, etc.) and these vibrations act in the same direction in all components. So gravitational waves cause relative changes in the

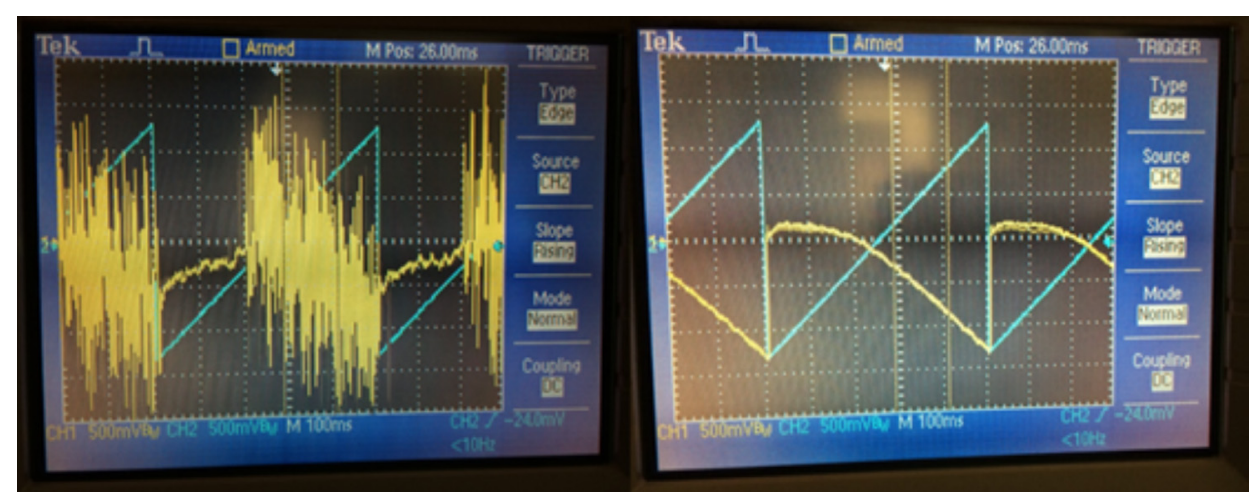

Fig. 14. Output of cavity locking circuit with positive (left) and negative (right) feedback. The sawtooth wave trace is the cavity PZT ramp, while the other trace is the feedback signal. 
distance between objects, while sound waves cause absolute changes in the objects' positions.

- For detecting gravitational waves, the test masses (the mirrors in the interferometer) need to be suspended so that they move freely in the horizontal plane. They are effectively in free fall at signal frequencies, since they are isolated from vibrations above $10 \mathrm{~Hz}$. Therefore, mechanical resonances are detrimental and need to be reduced as much as possible. In contrast, for sound wave detection, mechanical resonances in the test masses are desired since the detection actually works via their excitation.

- Even when coming from massive cosmic events, gravitational waves have extremely small amplitudes and are extremely difficult to detect. Sound waves are easy to detect and audible in a large frequency range.

\section{CONCLUSION}

It is obvious that the detection of gravitational waves is a delicate problem and that sophisticated methods are used to extract the tiny signal from an enormous background noise. As we have shown above, some of these methods and ideas can be demonstrated to students by presenting their fundamental operating principles in analogy experiments. We showed in several experiments how the sensitivity of a Michelson Interferometer can be increased by folding one or two arms with an optical cavity. We also demonstrated how this technique can be used in a sound wave experiment, with weak sound waves serving as the analog of gravitational waves. Furthermore, experimental problems such as finding the correct operating point and dealing with noise issues were addressed. Beyond the purely optical methods, we presented an advanced experiment on cavity locking, which involved sophisticated electronic techniques. By using a simple optical layout with homemade signal processing circuits for mixing and feedback control, an error signal was produced and used to lock the cavity. Finally, we noted some of the very fundamental and important differences between the real LIGO experiment and the presented lab experiments.

Since the physics of gravitational waves and their detection is complicated, and thus very challenging for undergraduate students, we hope that the presented set of experiments provides an easier access to these topics and offers better insights into the LIGO project, which has introduced a new era of experimental gravitational wave physics.

\section{ACKNOWLEDGMENTS}

This work was supported by the National Science Foundation under Grant No. PHY-1404269. This article has been assigned LIGO Document No. LIGO-P1800178. The authors also thank Thorlabs $\mathrm{GmbH}$ for providing setup components.

\section{APPENDIX: COMPONENTS LISTS}

In the following, we provide a list of the components we used for our experiments. We have not listed cables or other lab supplies, since these parts are usually available in any lab.
Table II. Basic components.

\begin{tabular}{lcccc}
\hline \hline & Manufacturer/ & & & $\begin{array}{c}\text { Total } \\
\text { Vest }\end{array}$ \\
\hline Equipment & Vendor & Part \# & Quantity & Thorlabs \\
Photodiode & HNLS008R-EC & 1 & $\$ 828.24$ \\
100k $\Omega$ resistor for & Thorlabs & SM05PD1A & 1 & $\$ 74.97$ \\
photodiode & Thorlabs & FT104 & 1 & $\$ 29.33$ \\
Converging lens & Thorlabs & LA1509 & 2 & $\$ 20.50$ \\
Mirror & Thorlabs & ME1-GO1 & 5 & $\$ 69.85$ \\
30:70 beamsplitter & Thorlabs & EBP1 & 1 & $\$ 32.90$ \\
50:50 beamsplitter & Thorlabs & EBS1 & 1 & $\$ 32.90$ \\
70:30 beamsplitter & Thorlabs & BST10 & 1 & $\$ 97.92$ \\
PZT & Thorlabs & PK2FQP2 & 2 & $\$ 187.68$ \\
Lens mount & Thorlabs & LMR1 & 5 & $\$ 76.15$ \\
Optical post & Thorlabs & TR2 & 12 & $\$ 62.28$ \\
Post holder & Thorlabs & PH2 & 12 & $\$ 92.40$ \\
Mounting base & Thorlabs & BA2 & 12 & $\$ 67.20$ \\
Mirror mount & Thorlabs & KM100T & 4 & $\$ 267.24$ \\
\hline \hline
\end{tabular}

Table III. Components for cavity locking.

\begin{tabular}{lcccc}
\hline \hline Equipment & $\begin{array}{c}\text { Manufacturer/ } \\
\text { Vendor }\end{array}$ & Part \# & Quantity & $\begin{array}{c}\text { Total } \\
\text { cost }\end{array}$ \\
\hline Power supply & Mean Well & RT-50C & 1 & $\$ 15.95$ \\
Function generator & GW Instek & SFG-1003 & 2 & $\$ 288.00$ \\
Demodulation chip & AD/Mouser & AD630 & 1 & $\$ 37.00$ \\
Switch & Digikey & 100SP1T1B1M1QEH & 2 & $\$ 4.22$ \\
$5 \mathrm{k} \Omega$ potentiometer & Newark & 652-3590S-2-502L & 2 & $\$ 27.36$ \\
$20 \mathrm{k} \Omega$ potentiometer & Mouser & 12V4NAYSD203A & 2 & $\$ 22.90$ \\
Utility enclosure & Hammond & 1411SS & 1 & $\$ 16.50$ \\
& Mfg. & & & \\
\hline \hline
\end{tabular}

Table IV. Components for sound experiments.

\begin{tabular}{|c|c|c|c|c|}
\hline Equipment & $\begin{array}{l}\text { Manufacturer/ } \\
\text { Vendor }\end{array}$ & Part \# & Quantity & $\begin{array}{l}\text { Total } \\
\text { cost }\end{array}$ \\
\hline USB oscilloscope & Pico technology & $\begin{array}{l}\text { PicoScope } \\
2000 \text { series }\end{array}$ & 1 & ca. $\$ 400$ \\
\hline PC loudspeaker & MusicMan & MusicMan mini & 1 & ca. $\$ 20$ \\
\hline
\end{tabular}

In Table II, the basic parts for setting up all optical components for the interferometers are shown. Of course, a (preferably damped) breadboard or optical table is required, however, we have no specific recommendations; any standard optical breadboard can be used.

Table III shows the additional components needed for the cavity locking experiments.

For performing the sound experiments, a USB oscilloscope with a spectrum analyzer function is required. Furthermore, a loudspeaker is needed for the sound signals. Table IV shows the parts we used. Of course, any similar equipment can do as well.

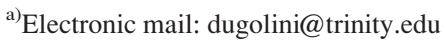

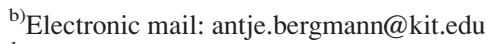

${ }^{1}$ B. P. Abbott et al., (LIGO Scientific Collaboration and Virgo Collaboration), "Observation of gravitational waves from a binary black hole merger,” Phys. Rev. Lett. 116, 061102 (2016).
} 
${ }^{2}$ A. Einstein, "Näherungsweise Integration der Feldgleichungen der Gravitation," Sitzungsberichte Königlich Preussischen Akad. Wissenschaften Berlin 1, 688-696 (1916).

${ }^{3}$ B. P. Abbott et al., (LIGO Scientific Collaboration and Virgo Collaboration), "GW170817: Observation of gravitational waves from a binary neutron star inspiral,” Phys. Rev. Lett. 119, 161101 (2017).

${ }^{4} \mathrm{~B}$. C. Barish and R. Weiss, "LIGO and the detection of gravitational waves," Phys. Today 52(10), 44-50 (1999).

${ }^{5} \mathrm{H}$. Mathur, K. Brown, and A. Lowenstein, "An analysis of the LIGO discovery based on introductory physics," Am. J. Phys. 85(9), 676-682 (2017).

${ }^{6}$ L. Burko, "Gravitational wave detection in the introductory lab," Phys. Teach. 55, 288-292 (2017).

${ }^{7}$ LIGO Scientific and VIRGO Collaborations, "The basic physics of the binary black hole merger GW150914,” Ann. Phys. (Berlin) 529(1-2), 1600209 (2017).

${ }^{8}$ Eric D. Black and Ryan N. Gutenkunst, "An introduction to signal extraction in interferometric gravitational wave detectors," Am. J. Phys. 71(9), 365-378 (2003).

${ }^{9}$ Eric D. Black, "An introduction to Pound-Drever-Hall laser frequency stabilization,” Am. J. Phys. 69(1), 79-87 (2001).

${ }^{10}$ J. Aasi et al., (LIGO Scientific Collaboration), "Advanced LIGO," Class. Quantum Grav. 32, 074001 (2015).

${ }^{11}$ A. A. Michelson and E. Morley, "On the relative motion of the earth and the luminiferous ether," Am. J. Sci. 34, 333-345 (1887).

${ }^{12}$ D. Herriott, H. Kogelnik, and R. Kompfner, "Off-axis paths in spherical mirror interferometers," Appl. Opt. 3, 523-526 (1964).

${ }^{13}$ P. R. Saulson, Fundamentals of Interferometric Gravitational Wave Detectors (World Scientific, River Edge, NJ, London, UK, 1994).
${ }^{14}$ A. Staley et al., "Achieving resonance in the Advanced LIGO gravitational-wave interferometer," Class. Quantum Grav. 31(24), 1-14 (2014).

${ }^{15}$ B. P. Abbott et al., (LIGO Scientific Collaboration and Virgo Collaboration), "GW150914: The advanced LIGO detectors in the era of first discoveries," Phys. Rev. Lett. 116, 131103 (2016).

${ }^{16}$ The LIGO Scientific Collaboration, "A gravitational wave observatory operating beyond the quantum shot-noise limit," Nat. Phys. 7, 962-965 (2011).

${ }^{17}$ D. E. McClelland, N. Mavalvala, Y. Chen, and R. Schnabel, "Advanced interferometry, quantum optics and optomechanics in gravitational wave detectors," Nat. Photon. Rev. 5, 677-696 (2011).

${ }^{18}$ B. P. Abbott et al., (LIGO Scientific Collaboration and Virgo Collaboration), "GW150914: Characterization of transient noise in Advanced LIGO relevant to gravitational wave signal," Class. Quantum Grav. 33, 134001 (2016).

${ }^{19}$ S. S. Y. Chua, B. J. J. Slagmolen, D. A. Shaddock, D. E. McClelland, (LIGO Scientific Collaboration and Virgo Collaboration), "Quantum squeezed light in gravitational-wave detectors," Class. Quantum Grav. 31, 183001 (2014).

${ }^{20} \mathrm{R}$. Abbott et al., "Seismic isolation for advanced LIGO," Class. Quantum Grav. 19, 1591-1597 (2002).

${ }^{21}$ D. V. Martynov et al., "Sensitivity of the advanced LIGO detectors at the beginning of gravitational wave astronomy," Phys. Rev. D 93, 112004 (2016).

${ }^{22} \mathrm{See}$ https://losc.ligo.org/events/ for data releases from all LIGO gravitational wave detections.

${ }^{23}$ AD630 Balanced Modulator/Demodulator Data Sheet. <http://www.ana$\log$.com/media/en/technical-documentation/data-sheets/AD630.pdf $>$

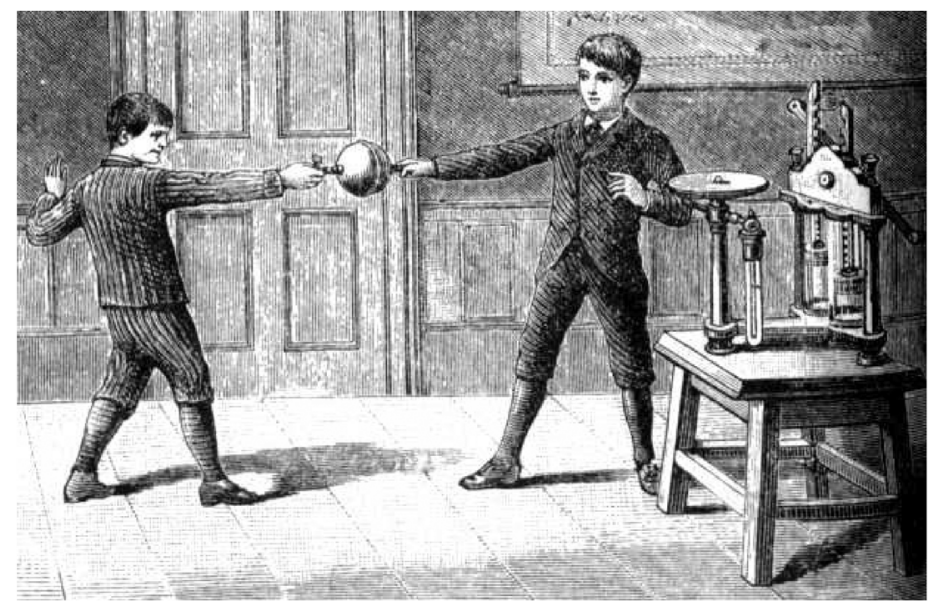

A Magdeburg Hemisphere Demonstration

When Otto von Guericke first did this demonstration in public about 1650, his hemispheres were 1.2 Magdeburgian ells in diameter (an ell is the distance from the elbow to the fingertips), and there were eight horses on one side and the same on the other. By the late years of the 19th century, when this figure was published in a secondary-level physics textbook, the hemispheres were smaller and the horses had been replaced by boys. But, the result was the same: the pressure differential of one atmosphere was enough to keep the hemispheres together. (Picture and Text by Thomas B. Greenslade, Jr., Kenyon College.) 